\title{
Prólogo
}

En mayo de 2011, en la ciudad de Toluca, México, en la Sesión de Sociedad Iberoamericana de Sistemas de Información Geográfica (SIBSIG) celebrada en el marco de la XIII Conferencia Iberoamericana de Sistemas de Información Geográfica (XIII CONFIBSIG), la Decana de la Facultad de Ciencias Espaciales de la Universidad Nacional Autónoma de Honduras asumió la Presidencia Ejecutiva de la Sociedad Iberoamericana de Sistemas de Información Geográfica, con el compromiso principal de organizar en la Universidad Nacional Autónoma de Honduras (UNAH), la Décimo Cuarta Conferencia Iberoamericana de Sistemas de Información Geográfica (XIV CONFIBSIG). A dos años de esa fecha, la Universidad Nacional Autónoma de Honduras fue la sede de esta Conferencia Iberoamericana que se desarrolló en la Ciudad Universitaria de Tegucigalpa, los días miércoles 3 , jueves 4 y viernes 5 de julio de 2013.

Las CONFIBSIG se han venido realizando de forma bienal y de manera ininterrumpida desde 1987, y como se ha venido repitiendo, se han llegado a definir como una de las reuniones científico tecnológicas de mayor alcance en Iberoamérica, dedicadas a delinear el estado del arte en cuanto a los desarrollos teóricos, metodológicos y de aplicación correspondientes a los avances realizados desde diferentes disciplinas que han encontrado en la Ciencia y las Tecnologías de la Información Geográfica, una dimensión fundamental para sus estudios.

La Décimo Cuarta CONFIBSIG, continuó ratificando esta definición y perspectiva gracias a las contribuciones de todos sus participantes provenientes de los países de Argentina, Brasil, Chile, Costa Rica, Cuba, Ecuador, España, Estados Unidos, Holanda, Honduras, México, Nicaragua, Panamá, Perú y Puerto Rico. Gracias también al esfuerzo de su Comité Científico Internacional integrado por los Doctores: Joaquín Bosque Sendra (España), Gustavo Buzai (Argentina), Delfino Madrigal Uribe (México), Julio Cesar Moraga (Costa Rica), José Seguinot Barbosa (Puerto Rico), Manuel Fuenzalida (Chile) y María Cristina Pineda de Carías (Honduras). Juntos ellos seleccionaron la ubicación de las comunicaciones incluidas por eje temático de la Conferencia. Gracias también a la dedicación y el empeño de los Miembros del Comité Local Organizador integrado por todo el personal docente del Departamento de Ciencia y Tecnologías de la Información Geográfica: Vilma Lorena Ochoa, Eduardo Moreno, Rafael Enrique Corrales, Liliam Sofía Gómez, Antonio 
Carías, Yeny Castellanos, Yessica Sosa, Jessica Villatoro, José David Cáceres y Celina Michelle Sosa, coordinados por el Decanato de la Facultad de Ciencias Espaciales. Se contó también con el apoyo institucional de la Rectoría, laVicerrectoría de Relaciones Internacionales, la Dirección de Investigación Científica y otras dependencias de la Universidad Nacional Autónoma de Honduras.

La Décimo Cuarta CONFIBSIG estuvo organizada en seis tipos de actividades:

- Dos Actos Especiales, uno de Inauguración para la presentación de la Conferencia; y el otro de Clausura para presentar las principales conclusiones.

- Seis Conferencias magistrales a cargo de reconocidos profesiona les de Argentina, Costa Rica, España, Honduras, México y Puerto Rico.

- Ochenta y tres Comunicaciones Orales, agrupadas por ejes temáticos, organizadas en veintidós sesiones dirigidas cada una por un Coordinador.

- Exhibiciones de empresas nacionales para mostrar el estado del arte en la aplicación de las tecnologías de la información geográfica.

- Una sesión de la Sociedad Iberoamericana de Sistemas de Información Geográfica.

- Incluyendo también un Acto Cultural en honor de todos los participantes.

La Décimo Cuarta Conferencia Iberoamericana fue precedida por tres cursos desarrollados, uno el 1 de julio, titulado "Identificación de Zonas de Potencial conflicto ante la evolución espacial de usos del suelo"; los otros dos, desarrollados el 2 de julio, titulados "Zonificación Territorial con SIG" y "Aplicaciones de los SIG en los estudios de cambio climático, la vulnerabilidad y la gestión del riesgo en países y ciudades de Centroamérica y el Caribe".

\section{CONFERENCIAS MAGISTRALES}

Las seis conferencias magistrales se programaron para ser la primera actividad de cada jornada, matutina o vespertina, de cada uno de los tres días de 
trabajo.

- En la mañana del día miércoles 3 de julio, el Dr. Julio Moraga (Costa Rica), como una Conferencia inaugural presentó el tema de "La inserción de la ciencia de la información geográfica en el currículo universitario", un asunto que desarrolló en el entorno de Costa Rica, destacando cómo ha evolucionado en la educación superior y en su difusión en las instituciones del país. La Ciencia de la Información Geográfica crecientemente se incorpora en la curricula, lo que ha motivado un proceso de actualización y capacitación continua por parte de los docentes. Los estudios de las demandas del mercado realizadas por las universidades para responder a la sociedad, se hicieron a través del seguimiento de los graduados, análisis de casos, de colocación, tareas realizadas, jornadas de trabajo entre otras, que permitieron identificar que un curriculum por competencias profesionales parece ser el más adecuado.

- En la jornada de la tarde, el Dr. José Seguinot Barbosa (Puerto Rico) presentó el tema actual del "Cambio Climático y vulnerabilidad de las comunidades residentes en la cuenca hidrográfica del Río Piedras, San Juan, Puerto Rico". Mediante una encuesta el determinó la percepción de los residentes sobre el ascenso del nivel del mar, la salinidad y las inundaciones y los efectos de estos parámetros sobre su salud, propiedad y calidad de vida. Esta situación la contrastó y modeló utilizando modelos de elevación, gráficos de líneas digitales y otros datos recogidos con un sistema de posición global, llegando también a determinar índices de vulnerabilidad para cada comunidad de la cuenca del Río Piedras. Destacó que en las investigaciones realizadas sobre cambio climático y vulnerabilidad, los Sistemas de Información Geográfica revelan la vulnerabilidad y el riesgo a que las ciudades pueden estar expuestas ante los cambios climáticos, y la importancia de que los gobiernos implementen políticas que reduzcan esa vulnerabilidad.

- El día jueves 4 de julio, tanto la conferencia de la mañana como la de la tarde estuvieron dedicadas al tema de los SIG aplicados a la seguridad ciudadana enfocados desde dos aproximaciones. Por la mañana el Dr. Delfino Madrigal Uribe (México) presentó conferencia sobre "Los Sistemas de Seguridad Pública basados en Sistemas de Información Geográfica". El explicó que de acuerdo al clima de violencia, al estado de la participación ciudadana, los niveles de consolidación de la autoridad gubernamental, así como el grado de disposición 
para combatir efectivamente el crimen, los SIG tienen diferentes estructuras y funciones. Que en la práctica debe entenderse que cada gobierno elige, de acuerdo a su grado de disposición, el grado de desarrollo del SIG. Un SIG altamente dinámico, interactivo y operativo debería integrar e interpretar todos los procesos y fenómenos involucrados, como base para la integración de políticas preventivas, correctivas y de combate efectivo al crimen.

- Por la tarde, la Rectora de la Universidad Nacional Autónoma de Honduras, Dra. Julieta Castellanos (Honduras), experta en temas de seguridad, abordó la temática desde la perspectiva de la "Seguridad ciudadana en Honduras". Ella presentó el caso del Observatorio Universitario de la Violencia, el cual ahora forma parte del Instituto Universitario en Democracia Paz y Seguridad de la Universidad Nacional Autónoma de Honduras. Explicó las diferentes etapas que ha pasado el Observatorio, desde su creación, organización, formas de trabajo, la utilización de sistemas de información geográfica, y su posicionamiento en la sociedad hondureña particularmente por la divulgación de boletines, mapas e indicadores de escala nacional, departamental, y en algunos casos municipal. De ambas intervenciones se concluyó que la seguridad ciudadana es un derecho de todo hombre, mujer, niño. Los dos investigadores pusieron de manifiesto como los SIG pueden generar elementos de juicio para orientar efectivamente los recursos del Estado para combatir la criminalidad. A través de los SIG se pueden identificar con mejor precisión los problemas y sus causas. Algunas estrategias podrían ser propuestas para ayudar al combate de la criminalidad, capacitación de cuerpos policiales y un manejo científico de las variables, concentrar esfuerzos en las áreas de mayor riesgo.

- El viernes 5 de julio por la mañana en una teleconferencia desde Argentina se presentó el tema de los "Sistemas de Información Geográfica en el contexto científico". El Dr. Gustavo D. Buzai sostuvo que los SIG han generado, al mismo tiempo, una revolución tecnológica que pone su atención en los métodos y técnicas de Análisis Espacial y, una revolución intelectual que la pone en las formas de estudiar y comprender la realidad centrándose en una focalización espacial. Presentó consideraciones sobre la globalización de la información en el sistema mundo para su análisis en escala humana.

- Por la tarde, la Dra. Montserrat Gómez Delgado (España), en la Conferencia de Cierre habló de "La necesidad de la validación en los procesos de Geosi- 
mulación" haciendo una revisión sobre la evolución que han experimentado los procesos de simulaciones espaciales aplicados a la planificación del territorio a partir de las TIG, derivando en la creación de una sub disciplina geográfica. Discutió sobre procedimientos adecuados de validación que avalen la fiabilidad y robustez de resultados de modelos que no pueden ser contrastados con datos reales, que pueden aminorar consecuencias negativas de las acciones del hombre sobre el territorio, ensayando procesos de planificación futuras más sostenibles.

\section{COMUNICACIONES TEMÁTICAS}

Ante los ejes propuestos por los organizadores de la XIV CONFIBSIG, los temas de las diferentes comunicaciones orales tomaron el peso de las preferencias y líneas de especialización de sus proponentes.

\section{Tema: Métodos y Análisis con Tecnologías de la Información Geográfica.}

Este tema tuvo el mayor peso con un $28 \%$ de las comunicaciones orales. En cinco sesiones distribuidas en los tres días de la Conferencia, los comunicadores expusieron sobre el crecimiento urbano estudiado con técnicas de modelaje con autómatas celulares en SIG, en pequeñas ciudades, una región o una comunidad, hasta incluir todo un país, llegando a abordar hasta desequilibrios urbanos por contaminantes ambientales. En este tema se presentaron análisis de detección de cambios de la cobertura y uso del suelo de períodos desde diez hasta veinticinco años, incluyendo técnicas estadísticas para detección de cambios importantes y procesos sistemáticos. Análisis de variables socioeconómicas, econometría espacial y geoestadística; inteligencia de negocios; y efectos ambientales de actividades humanas estudiados con técnicas de estimadores de densidad de núcleo también fueron expuestos. Las anomalías espectrales con implicaciones terrestres en la exploración planetaria, identificación de formaciones geomorfológicas características de cráteres, y el uso de lógica fuzzy en modelos probabilísticos de ocurrencia de deslizamientos fueron discutidos. SIG y evaluación multicriterio para apoyo de gobiernos locales, y software libre para gestión y planificación de recursos hídricos fueron presentados. Localización y reconocimiento de sitios arqueológicos mediante técnicas no intrusivas, llegando hasta la búsqueda de algunos con significado arqueoastronómico. Técnicas de posicionamiento global para determinación de una superficie correctora para modelado de un geoide; incluyéndose también en este 
grupo de comunicaciones un marco teórico sustentado en la teoría de la geografía en la búsqueda de resultados concretos a través de una ciencia aplicada.

\section{Tema: Recursos Naturales, Ambiente y Cambio Climático.}

Este tema abarcó el $25 \%$ de las comunicaciones. En cinco sesiones que se desarrollaran a lo largo de los tres días, se presentaron estudios sobre fragmentación de ecosistemas a partir de cálculo de métricas de paisaje, evaluación de cambios de áreas de bosque nativo y su relación con situaciones de vulnerabilidad ambiental, identificación de zonas propensas a desertificación, análisis de vulnerabilidad ante el impacto de desastres y amenazas antrópicas, naturales y socio naturales, cambios en la cobertura del terreno por contaminación ambiental y sobre explotación de recursos, dinámicas de cambio, y coberturas de ecosistemas prioritarios como arrecifes, pastos marinos y manglares. Se discutieron estudios de acuíferos por procesos de subsidencia a partir de técnicas de interferometría diferencial para estimar deformaciones del terreno en una cuenca; el uso de geotecnologías para monitoreo y evaluación de recursos naturales en glaciares y ríos de hielo; análisis multitemporal de cambios de uso del suelo de cuencas y dinámicas de cambio por períodos de hasta veinte años; degradación de la calidad del agua e impacto agrícola determinado por análisis multicriterio; y estudios geoestadísticos de análisis de variables climáticas como la precipitación y la temperatura. La estimación de biomasa y stocks de carbono en cubiertas herbáceas, y la cuantificación de biomasa y carbono forestal para varios tipos de bosque fueron discutidos; así como estudios de incendios forestales para determinación de riesgos de espacios protegidos, y análisis de causas de modificación de ambientes entre llanuras y parques por la probabilidad de ocurrencia de incendios. Dinámica de cambios de la cobertura vegetal por actividades petroleras utilizando técnicas de autómatas celulares para el modelamiento espacial de la cobertura. Mapeo exploratorio con LIDAR para revelar lo que el bosque oculta, como una hidrografía compleja y una extensa modificación antropogénica que parece ser restos arqueológicos. Muestreos de aves en extinción en diferentes hábitats, y la Internet del Futuro, Internet de las cosas y del medio ambiente también fueron discutidos.

\section{Tema: Cartografía, Catastro y SIG Municipales.}

Este tema abarcó el $12 \%$ de las comunicaciones de la Décimo Cuarta CONFIBSIG. Incluyó tres sesiones en las que se presentaron procedimientos para ela- 
boración de mapas de zonificación territorial aplicada a municipios, y la elaboración de cartografía de uso y cobertura del suelo con metodologías de clasificación visual de imágenes satelitales y comparación con series cartográficas de años anteriores. La elaboración de atlas geográfico y satelital como herramienta interactiva con información confiable y de fácil acceso; Atlas de información territorial de unaRegión; Atlas de riesgos de fenómenos naturales con visualizaciones del tiempo desde perspectivas teórico prácticas; uso de SIG y mecanismos de visualización para popularización del ambiente urbano y el patrimonio histórico, cultural y urbano; y la comparación de si la cartografía multimedia con alto nivel interactivo es más útil que los medios convencionales para la comunicación de patrimonio de centros históricos. Se discutió la calidad en la cartografía mediante el uso de cámaras fotogramétricas, redes geodésicas nacionales, y aerotriangulación cinemática; y el monitoreo de deformaciones superficiales sobre fallas geológicas activas mediante técnicas geodésicas satelitales y análisis matemáticos y estocásticos para obtener información de calidad.

\section{Tema: Ordenamiento Territorial.}

Este tema igualmente abarcó el $12 \%$ de las comunicaciones de la XIV CONFIBSIG. Se desarrolló en tres sesiones para presentar una metodología aplicada al municipio que facilita la elaboración de escenarios de prospectiva para asentamientos humanos, en suelos rural y urbano, con el fin de planificar su crecimiento con garantías de bienestar. Estrategias de desarrollo municipal mediante estudios que abarcan desde viabilidad técnica y económica hasta la adquisición del suelo, la gestión, el diseño y la ejecución de la actuación urbanística para el desarrollo de actividad económica. Se presentó una propuesta de análisis de sensibilidad explícitamente espacial, utilizando herramientas de un SIG aplicado a la simulación del crecimiento urbano futuro de una comunidad, realizando una asignación óptima de suelo residencial, industrial y comercial para el término de veinte años. Se analizó el papel de la ordenación del territorio en la construcción de lugares saludables, ante problemas ambientales y socioeconómicos que inciden negativamente en la salud de la población. Se presentaron estudios de asentamientos en laderas y, temas de riesgos y morfología urbana en planicies y laderas. Se discutió la utilización de un SIG de gestión integral de tierras para apoyar la descentralización del mantenimiento catastral y la aplicación de planes de ordenamiento territorial por autoridades locales para garantizar la actualización continua de datos de tenencia y uso de la 
tierra; así como también la generación de una base de datos para el archivo técnico de la Dirección General de Catastro y Geografía ligando fotografías aéreas a hojas cartográficas. Se presentaron instrumentos necesarios para la construcción de un SIG como apoyo a la gestión de centros históricos; y un modelado 3D y recorridos de realidad virtual interactivos sobre un SIG referido al estado de conservación arquitectónica de las principales edificaciones de la Ciudad Universitaria.

\section{Tema: Formación y Enseñanza en Tecnologías de la Información Geográfica.}

Este tema comprendió el $8 \%$ de las comunicaciones. Organizado en dos sesiones se presentaron resultados de una revisión de los estudios de TIG que en la actualidad se imparten en España y Europa, en grados y másteres; analizando sus principales características, objetivos, duración, contenidos de asignaturas, hasta llegar a formular una primera clasificación para conocer como se insertan y enseñan estas tecnologías. Se planteó como surgió en Honduras el desarrollo de los SIG ante una demanda de profesionales expertos después de una catástrofe natural, y como organismos internacionales colaboraron con instituciones gubernamentales creando info-sistemas y capacitando técnicos sin una base sustentable que trajo como consecuencia la generación y el manejo empírico de datos geoespaciales, dándose ahora condiciones para una formación planteada desde una perspectiva general, directiva, técnica y estudiantil. Se describieron las primeras iniciativas para el establecimiento de las TIG en la UNAH, el surgimiento de la Maestría en Ordenamiento y Gestión del Territorio con la cooperación desde un inicio de la Universidad de Alcalá, sus principales líneas de investigación y del campo profesional de sus primeros graduados, poniendo en este contexto de desarrollo las iniciativas de formación a nivel de asignaturas generales y el planteamiento de una nueva carrera de grado con salidas laterales para la formación de técnicos. Se planteó una lista de competencias a alcanzar por los estudiantes que realicen algún estudio universitario en SIG, haciendo una evaluación y discusión crítica para generar una propuesta propia de las competencias más importantes y necesarias para obtener un título de SIG. Se analizaran problemáticas y retos geoéticos en un contexto docente del ordenamiento y la gestión del territorio, aspectos relacionados con códigos de conducta y buen hacer que ha tenido que abordar el profesorado nacional e internacional de la Maestría de Ordenamiento y Gestión del Territorio en sus sucesivas promociones, realizando una aproximación desde distintas áreas del conocimiento para el tratamiento conceptual profesional y de investigación de la Geoética en un ámbito Centroamericano, para aportar respuestas ante nuevos retos globales. Se 
plantearon las formas y metodologías aplicadas por la Maestría de Ordenamiento y Gestión del Territorio en su proceso de acreditación regional haciendo una crítica reflexiva y colectiva de ocho componentes: estudiantes, graduados, profesores, proceso formativo, investigación e innovación, gestión académica y administrativa, vinculación, proyección e incidencia social y colaboración e intercambio académico nacional, regional e internacional. Se señaló la importancia de los atlas digitales en el proceso de formación y enseñanza, con el propósito de que el estudiante tenga como base los principios de la Geografía, localización, distribución, causalidad, multicausalidad y cambios para diversas aplicaciones a problemas reales.

\section{Tema: Salud y Seguridad Alimentaria.}

Este tema ocupó el 7\% de las comunicaciones. Los comunicadores destacaron la importancia de los SIG para determinar los patrones de distribución de tasas de mortalidad en un período de treinta años y generar escenarios a veinte años a nivel local y municipal, utilizando fuentes del sistema nacional de información en salud y anuarios estadísticos; y un diagnóstico actual de la localización de los servicios públicos de salud primarios, en relación a su estructura, dinámica y relaciones en una zona metropolitana, y al mismo tiempo la elaboración de una propuesta desde el punto de vista de los factores necesarios para la localización óptima de dichos servicios. Se presentó también un panorama global y actualizado de la relación entre el uso de los SIG y los Sistemas de Ayuda a la Decisión Espacial (SADE) y la Geografía de la Salud, una de las líneas de investigación con mayores posibilidades de aplicación en el estudio de distribuciones espaciales. Se planteó un estudio para conocer el estado de las emisiones del ruido en la ciudad, partiendo de la determinación de sitios de muestreo en base a la densidad del tráfico, el registro de las mediciones del ruido ambiental, evaluando la información con el método estadístico de Kriging ordinario para llegar a elaborar mapas de ruido de la ciudad. Con este mismo método geoestadístico se presentó un modelo de la exposición de contaminantes atmosféricos MP10 a partir de los datos registrados en ciento ocho estaciones de monitoreo pertenecientes al sistema nacional de calidad del aire. Se presentaron análisis de los riesgos físicos ocurridos en los últimos veinticinco años y su impacto en la salud pública, utilizando un SIG para analizar distribuciones espaciales y temporales por regiones de salud y por municipio. 


\section{Tema: Infraestructura de Datos Espaciales.}

Este tema ocupó el 7\% de las comunicaciones. En las sesiones se planteó como en Honduras, la Infraestructura de Datos Espaciales (INDES) está regulada por la Comisión de Datos Espaciales, y que para impulsar su desarrollo se ha iniciado un largo proceso que ha requerido de una voluntad política, generación de capacidades, un voluntariado institucional y un presupuesto, y de la integración de un organismo nacional de normalización que ha trabajado en base a las normas ISO relacionadas con los metadatos. Se describieron las principales acciones metodológicas llevadas a cabo para la implementación de una Infraestructura de Datos Espaciales (IDE) regional, a partir de las experiencias en el Laboratorio de SIG de la Universidad, que en referencia a los estándares ha optado por el perfil del metadato de la propuesta desarrollada por el Instituto Geográfico Agustín Codazzi denominado Perfil Latinoamericano de Metadatos. Se planteó una metodología para la creación de una geodatabase que permitió ordenar archivos de la elaboración digital de hojas cartográficas, mapas departamentales, mapas municipales, mapa oficial del territorio de Honduras y otros generados por la Dirección General de Catastro y Geografía. Se planteó que la elaboración o actualización de la cartografía nacional de un país debe iniciarse con el análisis pormenorizado de las opciones disponibles, el Instituto Geográfico Nacional de España expuso que se dispone de una gran variedad de productos cartográficos básicos y derivados que cubren una gran variedad de escalas, y que en algunas series se dispone de una dualidad entre la base de datos de información geográfica y la versión mapa o cartográfica de la misma, pudiendo de esta forma conservarse aproximadamente mitad en la base de datos y en el soporte de la base topográfica nacional. Se expuso sobre el montaje de un servicio de mapas en la web con la utilización de software libre para compartir información geoespacial del país, y que ésta a su vez sea de fácil localización, amigable y que esté regulada por la Dirección General de Catastro y Geografía. Se mostró también la utilización de tecnología geoespacial para la demarcación de las fronteras Honduras-México y Honduras-Cuba.

\section{PRINCIPALES CONCLUSIONES}

Las conclusiones fueron redactadas por los Coordinadores de las Sesiones de los diferentes ejes temáticos, correspondiendo a la Coordinación General su edición. Las principales conclusiones por tema de la XIV CONFIBSIG fueron las siguientes: 


\section{Tema: Métodos y Análisis con Tecnologías de la Información Geográfica.}

- Resultan de especial interés los esfuerzos realizados para la simulación del crecimiento urbano, con diferenciación de asentamientos cerrados y periféricos, mediante técnicas de autómatas celulares, considerando patrones ambientales de vecindad y difusión.

- Se han podido identificar cambios principales en los bosques, en los pastos con transiciones de agricultura por la evaluación del cambio del uso del suelo en el Parque Nacional Miguel Hidalgo y Costilla La Marquesa (1994-2007), aplicando tecnologías de SIG.

- Existen varios métodos estadísticos para verificación de los resultados, que mediante análisis multitemporal de teledetección espacial y SIG aplicados al cambio de la cobertura de suelo, permiten asegurarnos que se apeguen a la realidad.

- El método de Pontius es una nueva forma de explicar los datos estadísticos a partir de matrices de tabulación cruzada para calcular persistencia, pérdidas, ganancias, cambio neto, intercambio y transiciones sistemáticas. Se ha podido comprobar que más del $50 \%$ de un sector del occidente de Honduras ha experimentado un alto dinamismo del paisaje entre las categorías de Bosque Mixto a bosque de pino y de Matorral a Agricultura antes del Huracán Mitch; y de bosque de pino a Bosque Mixto y de Matorral a Bosque de pino después del huracán.

- Con la aplicación de imágenes interespectrales es posible construir un mapeo de las alteraciones presentes en áreas volcánicas, sobre todo para restablecer la precisión determinadas metodologías y técnicas.

- El uso de imágenes y tratamientos cruzados de filtros de bordes y técnicas Crosta permiten encontrar las cicatrices de eventos geológicos tales como cicatrices de cráteres, indetectables a simple vista.

\section{Tema: Recursos Naturales, Ambiente y Cambio Climático.}

- En el tema de recursos naturales y cambio climático, se pueden realizar estudios utilizando los mapeos mediante datos ópticos y radares integrales en el uso de SIG, además se pueden realizar análisis de cobertura y usos del suelo, mediante la utilización de las imágenes satelitales y técnicas metodológicas aun de bajo costo.

- Las comunicaciones se enfocaron a la definición de condiciones de vulnera- 
bilidad en distintas ecorregiones, en un caso a partir de la definición de parámetros morfométricos, que serán parte de los insumos en la generación de los SIG, por otro lado a partir de desarrollos en comunidades de Costa Rica, que permitirán conocer escenarios de riesgo y delinear acciones de prevención, mitigación y monitoreo.

- Se destacó la generación de un SIG que permite a través de sensores LIDAR, monitorear áreas con alto valor arqueológico tales como en la región de la Mosquitia hondureña, con miras a la preservación de las mismas.

- La utilización de los sensores multiespectrales permite definir la batimetría en la laguna de Sierpe ayudando al monitoreo de condiciones hidrológicas.

- En el campo del cambio climático resaltan los esfuerzos para la cuantificación de los stocks de carbono en estratos herbáceos y forestales, implementando técnicas de teledetección cercana y remota, así como la automatización de los métodos de procesamiento, empleando sensores de media y alta resolución espacial.

- Se ha podido desarrollar un programa de accesibilidad y común para cualquier usuario en el mundo que proporciona servicios genéricos de ubicación, temperaturas, entre otros.

- Se ha podido desarrollar un estudio que demuestra la fragmentación de bosque de gran tamaño a pesar de la intervención humana y cultivos de exportación.

- La utilización de los SIG es de gran utilidad para las técnicas estadísticas y geo estadísticas en la aplicación en los territorios y especialmente para las precipitaciones.

- Existe relación variable de los puntos de calor versus caminos, teniendo en cuenta que los datos no son aún respaldados con trabajo de campo, se incluirá otras variables y otras áreas para probar la metodología.

- Mediante el uso de la clasificación no supervisada, se dificulta reconocer las diferencias entre clases de suelo por conglomerados fisiológicos.

- El análisis multicriterio de Sub cuenca como la del Rio Mocal determinará el impacto de la actividad agrícola teniendo variables como análisis de agua y análisis socioeconómicos que demuestran la afectación de los contaminantes ante el aumento del caudal en la Sub Cuenca del Río Mocal.

- El sistema de Autómatas Celulares, es una herramienta importante para la determinación de análisis prospectivo con ayuda de sistemas de información geográfica y Teledetección a través de las fotografías aéreas, para el modelamiento del cambio del uso del suelo y cobertura vegetal. 
- Se aplican en diversos campos como ser: simular el crecimiento poblacional, desplazamientos, Ordenamiento Territorial, Riesgos naturales, Cobertura Vegetal y Uso del Suelo.

\section{Tema: Cartografía, Catastro y SIG Municipales.}

- Los SIG son importantes instrumentos para realizar una preservación patrimonial y ambiental, como es el caso de los Inventarios y los Atlas.

- La educación puede encontrar importantes apoyo en los SIG para la enseñanza formal, de secundaria y para el uso de la comunidad.

- Es importante abarcar más análisis espaciales como los catastros y SIG municipales, aproximando los trabajos a los resultados para futuras ciudades.

- Existen mecanismos dinámicos a través de los símbolos para representar fenómenos en el tiempo.

\section{Tema: Ordenamiento Territorial.}

- La representación geográfica en 3D con su interrelación con los SIG, sigue ganando terreno en los procesos de ordenamiento territorial y gestión del patrimonio tangible. La visualización de este tipo da la posibilidad de analizar propuestas de desarrollo urbano y su relación con el contexto, paisaje y crecimiento de la infraestructura física de un lugar, de una forma más comprensible.

- Se reconoce que el proyecto que la Secretaria de Educación Pública de Honduras ha hecho referente al levantamiento, descripción y análisis del estado y contribución de los centros educativos mediante Sistemas de Información Geográfica está refrendando la potencialidad que estas herramientas tienen para mejorar la administración pública y la distribución de los recursos con que se cuentan.

- Metodologías de zonificación territorial con Sistemas de Información Geográfica contiene lineamientos metodológicos básicos para la aplicación de los SIG en la generación de mapas de zonificación nacional, regional y municipal, siguiendo un enfoque multinivel que asegura la coherencia entre los diferentes de planificación territorial.

- En ordenamiento territorial, son muy importantes los análisis de uso del suelo y prospectivas tanto en asentamientos urbanos como rurales, resaltando la necesidad de disponer de bases de datos geográficos para la planificación y gestión del territorio y sobre las características morfológicas de los asentamientos 
humanos.

- Se ha podido mostrar el papel que desempeña el ordenamiento territorial en la salud de la población, tomando como universo de estudio la zona metropolitana de Toluca. La metodología utilizada contiene variables e indicadores, ambientales, culturales sociales y su relación con la saludad de la población que habita ese espacio. Como conclusiones presenta un enfoque holístico de la salud, enfatizando que para el bienestar de la población los factores: físicos, síquicos, ambientales y sociales juegan un papel importante y que tiene que ver con el territorio en el que habitan. Por lo anterior se promueve a que las políticas orientadas a mejorar la salud de la población sean focalizadas y no generales y que se incluyan en los planes de desarrollo urbano, con mayor vinculación hacia la sociedad.

- Se presentó una propuesta metodológica de herramientas para poder manejar los centros históricos que permitan a cualquiera gestionarlos, haciéndose énfasis en la utilización de sistemas de información geográfica combinadas con la gestión del patrimonio. Estas pueden ser: estructura urbana de la ciudad, estructura de base de datos, datos cartográficos, decretos, etc. Como conclusión se estable que para la gestión de centros históricos es necesario tener herramientas legales y técnicas, explotar la extracción de datos, así como el uso de herramientas económicas.

- Sobre el levantamiento de datos y generación de mapas temáticos y su aplicación a técnicas participativas de la población para lograr el desarrollo urbano de una colonia de Tegucigalpa que es poblada por medio de la invasión, como en el país falta información geoespacial es evidente, este proyecto muestra la forma en que el Instituto Hondureño de Ciencias de la Tierra de la UNAH, aporta a esta comunidad y al proyecto urbano la información física y topográfica de la zona, utilizando tecnologías de GPS. Como conclusión se presenta la falta de datos espaciales en Honduras que no permite el desarrollo de proyectos de tipo territorial, pero que en la UNAH se ha aportado usando TIG.

- Se presentó un atlas de la Pampa Argentina, cuyo objetivo es presentar y conocer el territorio, desplegar información actualizada recopilando la mayor cantidad de datos posibles del territorio, y con la idea de que las personas que lo utilicen puedan generar nuevos datos a partir de estos. Se utilizan imágenes satelitales y el servicio es de uso público con énfasis en la educación secundaria.

- Se presentó comunicación centrada en la ciudad como un artefacto cultural para la preservación del patrimonio de la ciudad de Pelotas. Para representar el patrimonio se utilizan tres escalas distintas de visualización, las cuales toman 
distintas unidades de visualización: el lote, el volumen y la edificación. Concluyendo que los SIG (publicación en la Web) y la preservación del patrimonio deben ir ligados para fortalecer su gestión y elaboración de políticas públicas, porque permiten ver el todo integralmente.

- Se presentó Atlas multimedia del Centro Histórico del Distrito Central de Honduras, que se orienta a aplicarse a un usuario en específico, con la idea de transmitir la importancia de la preservación de un centro histórico. El atlas es un producto multimedia que muestra de manera interactiva el desarrollo del centro histórico de la ciudad de Tegucigalpa, se aplican pruebas a posibles usuarios para comprobar su uso frente a otros tipos de cartografía más convencional (atlas de papel). Se concluye que el uso de la multimedia beneficia más en cuanto a tiempo de uso, capacidad de seleccionar información, y de mostrar información espacial detallada de la zona de estudio.

\section{Tema: Formación y Enseñanza en Tecnologías de la Información Geográfica.}

- En el gran contexto de la formación y enseñanza de las TIG, nos encontramos con el gran dilema de las ambigüedades sobre la temática respectiva en los grados académicos, desde técnicos hasta doctorados. Es por ello que surgen las preguntas de como dosificar las clases para que un grado no interfiera con otro; como orientar un grado académico hacia las nuevas tecnologías de programación web, que es el paso que estamos viviendo ya que todo recurso personalizado tiene un componente de programación muy avanzado lo cual aumenta la carga del plan que se está estudiando, esta relación entre la dinámica tecnológica que nos lleva de la mano a un paso muy acelerado y constante cambio evolutivo en los sistemas geográfico y en los propios planes de estudio, los cuales se vuelven cada vez más robustos y complejos, al querer abarcar toda la temática multidisciplinaria, por lo que muchas iniciativas intentan mejor incluir en sus planes de estudios un fuerte componente en TIG para realizar el análisis espacial con la cosmovisión de la temática de estudio.

\section{Tema: Salud y Seguridad Alimentaria.}

- Los principales determinantes de mortalidad en la zona metropolitana de Toluca son: diabetes mellitus y enfermedades isquémicas de corazón. El análisis geoestadístico es una herramienta que facilita la generación de mapas de tendencias de salud para el desarrollo de proyectos dirigidos a comunidades 
vulnerables, establecer normas y estrategias para disminuir los riesgos entre estas enfermedades y bajar las tasas de mortalidad.

- La aplicación de las teorías de distribución por justicia social y espacial de salud pública, permite encontrar una mejor localización de centros hospitalarios a través de múltiples criterios y mostrar a nivel de diagnóstico las necesidades reales en el sistema de salud.

- Desde las diferentes aplicaciones temáticas para representar los aspectos de salud, podemos mostrar un diagnóstico rápido de cómo se encuentra un sistema de salud de un espacio determinado, y con estas técnicas de evaluación multicriterio para generar una distribución con equidad espacial para nuevos puntos sanitarios, mejorar los sistemas actuales de distribución sanitaria.

- Ya que el ruido afecta la salud y la economía, es indispensable medir los niveles de ruido en una ciudad o en sitios cercanos a hospitales, por lo que con la aplicación de métodos de inverso de las distancias ponderadas y con un Kriging ordinario se logra modelar la dinámica del ruido y establecer que zonas son las que evidentemente tienen problemas por ruido y generar o actualizar normativas para el control del ruido en horas pico, sea de día o de noche.

- Los temas de seguridad se pueden utilizar para realizar estudios de impacto en el área de la salud a partir de la ocurrencia de desastres naturales.

\section{Tema: Infraestructura de Datos Espaciales.}

- La calidad cartográfica se logra a partir de datos ordenados y confiables, es así que hoy en día existen estándares ISO, que certifican las infraestructuras de datos espaciales.

- La infraestructura es el motor para que funcione una adecuada operatividad entre los diferentes departamentos institucionales.

- Las tecnologías de SIG utilizadas para generar Cartografía, vienen a acelerar procesos permitiendo un mejor flujo de trabajo, en menor tiempo, además influyen en el desarrollo del país.

- La infraestructura de Datos Espaciales IDE se hace imprescindible en el proceso de almacenamiento de información espacial, así como en la gestión de la información desde su producción hasta la publicación.

- En la recopilación y sistematización de datos espaciales, existen en las series cartográficas y bases de datos, surgen algunos problemas y debilidades para la producción de mapas derivados de la generalización en el abanico de las escalas. 
- Las infraestructuras de datos espaciales facilitan el acceso a todo tipo de información relacionado con la recopilación y sistematización de Datos Espaciales, logrando mapas de muy buena calidad tanto métrica como numérica.

\section{SESION DE LA SOCIEDAD IBEROAMERICANA DE SISTEMAS DE INFORMA- CIÓN GEOGRÁFICA}

Los Estatutos de la Sociedad Iberoamericana de Sistemas de Información Geográfica (SIBSIG) tienen establecido que, al final de cada Conferencia Iberoamericana de Sistemas de Información Geográfica se realice una Sesión con todos los participantes como miembros.

En la ciudad de Tegucigalpa, el día cinco de julio de dos mil trece, en el Auditórium Central "Juan Lindo" de la Ciudad Universitaria de la Universidad Nacional Autónoma de Honduras, se celebró la Sesión de la Sociedad Iberoamericana de Sistemas de Información Geográfica en la que se desarrolló la siguiente Agenda:

Punto 1. Apertura de la Sesión. El Presidente General de la SIBSIG, Dr. Delfino Madrigal Uribe, declaró abierta la Sesión Plenaria de la Sociedad Iberoamericana de Sistemas de Información Geográfica. Expresó que se había estado platicando con la Presidenta Ejecutiva y los expresidentes, y que juntos habían acordado presentar a consideración de la Asamblea la Agenda propuesta. Declaró también que una cosa importante que se había estado platicando con los expresidentes y la Presidenta Ejecutiva era la necesidad de poder llevar a cabo diferentes líneas de trabajo que le den mayor consistencia a la SIBSIG, para darle mayor presencia, sobre todo para que los jóvenes y estudiantes de posgrado tengan la oportunidad de seguir estos esfuerzos. Preguntó que si no existía observación a la Agenda se procedería al traspaso de la Presidencia General.

Punto 2. Traspaso de la Presidencia General de la SIBSIG. De conformidad con los Estatutos de la SIBSIG, la Presidencia General es asumida por un período de dos años por la persona que se ha desempeñado como Presidente Ejecutivo de la SIBSIG. En este sentido, el Dr. Delfino Madrigal Uribe, Presidente General de la SIBSIG 2011-2013 traspasó la Presidencia General a la Dra. María Cristina Pineda de Carías, para asumir el cargo por el Período 2013-2015. La Dra. Pineda de Carías agradeció al Dr. Uribe, y aclaró que en el nuevo cargo que asumía no estaba sola, que estaba acompañada de todo un equipo y un Consejo Asesor de todos los 
Expresidentes de la SIBSIG que nos acompañaban.

Punto 3. Informe y ratificación de candidatura de Universidad Católica de Valparaíso, Chile, para ser sede de la XV CONFIBSIG-2015. Ya en posesión de su cargo, la nueva Presidenta General informó que además de la candidatura de la Pontificia Universidad Católica de Valparaíso, Chile, no se había recibido ninguna otra candidatura. Pidió entonces a todos los presentes constituidos en Sesión que para ratificar la candidatura, se debía escuchar la propuesta de parte del Dr. Manuel Fuenzalida en representación de la Pontificia Universidad Católica de Valparaíso, Chile. El Dr. Fuenzalida dijo que traía la Carta del Rector de la Universidad en la que ratificaba el patrocinio a la postulación del Instituto de Geografía como sede de la XV CONFIBSIG a realizarse en el año 2015, e inmediatamente entregó la Carta de Patrocinio. Formalmente recibida la ratificación del Rector de la Pontificia Universidad Católica de Valparaíso, y cumplidos los requisitos de Ley, la Presidenta General pidió a todos los presentes levantar la mano para ratificar la candidatura. Por unanimidad se aprobó la propuesta de la XV CONFIBSIG en Valparaíso, Chile, en 2015.

Punto 4. Traspaso Presidencia Ejecutiva de la SIBSIG. Después de haber sido aceptada la sede de la XV CONFIBSIG en Valparaíso, Chile, la Presidenta General pidió al Dr. Manuel Fuenzalida que pasara a la mesa principal. La Dra. Pineda inmediatamente traspasó la Presidencia Ejecutiva de la SIBSIG al Dr. Fuenzalida, expresándole que su compromiso principal era dirigir la organización de la XV Conferencia Iberoamericana de Sistemas de Información Geográfica con sede en Chile. Invitó a todos los participantes a poner en sus agendas esta nueva conferencia del año 2015.

Punto 5. Solicitud de candidaturas para sede de la XVI CONFIBSIG 2017. Definida la sede de la XV CONFIBSIG para 2015, y siguiendo procedimientos establecidos por la SIBSIG, la Presidenta General preguntó a los presentes que después de la actividad de Chile, quien sigue, qué Universidad o qué Institución tiene el interés, o trae la solicitud formal para presentar la candidatura de ser la sede de la XVI CONFIBSIG para el año 2017. El Dr. Omar Delgado de Ecuador, pidió la palabra para presentar formal solicitud para poder organizar la XVI CONFIBSIG en Ecuador, específicamente en la Universidad del Asuay, en la ciudad de Cuenca. Para ello, dio lectura a comunicación dirigida a la Presidenta de la SIBSIG, en la que planteó y puso en consideración la candidatura de la Universidad del Asuay, a través del Instituto de Estudios de Régimen Seccional del Ecuador IERSE, adscrito al Deca- 
nato Federal de Investigaciones como sede de la XVI CONFIBSIG a realizarse en el año 2017. "Quiero dejar constancia que los compromisos a ser adquiridos como sede candidata a la XVI CONFIBSIG serán asumidos con responsabilidad a través de una adecuada y oportuna gestión académica, administrativa y financiera para la organización del evento. Atentamente, Omar Delgado, Director del Instituto de Estudios de Régimen Seccional del Ecuador". Leída la carta, ésta fue entregada a la Presidenta General de la SIBSIG quien dio por recibida la candidatura de la Universidad del Asuay de Ecuador. Siguiendo el curso de la Sesión, hizo la pregunta a los presentes de si había otra candidatura. No habiendo más candidaturas respondió al Dr. Delgado que al dar por recibida la propuesta, y que cuando nos encontremos en la XV CONFIBSIG en Chile, hay que ratificar la candidatura porque el procedimiento así lo exige.

Punto 6. Líneas de Trabajo SIBSIG - Período 2013-2015. Para presentar las Líneas de Trabajo, la Presidenta General de la SIBSIG comentó que estas líneas las habían venido trabajando en estos últimos días con los expresidentes de la SIBSIG. No es un Plan de Trabajo formal desde luego, porque eso requerirá de más tiempo, y desde luego la participación de todos la cual pidió en ese momento.

- Apertura página web oficial de la SIBSIG. En esta CONFIBSIG se ha incluido la virtualidad, que la imponen los tiempos y las formas de comunicación a través de la red. Entonces, el primer compromiso es abrir la Página web oficial de la Sociedad Iberoamericana de Sistemas de Información Geográfica (SIBSIG). Un sitio que ya va a quedar formalmente continuado porque hasta ahora ha venido manejándose aisladamente según la Universidad que ha asumido la CONFIBSIG. La idea es tener una página que pueda tener continuidad, por lo que oportunamente se les enviará la comunicación a cada uno de Ustedes, para entre todos irla mejorando.

- Organización. Comisiones. La información básica que nos vamos a estar comunicando en esa página web, en primer lugar es la Organización de la Directiva que tenemos, saber quiénes somos Presidente General, Presidente Ejecutivo, y los diferentes cargos que puedan ir saliendo en la medida que la organización vaya tomando una forma más amplia, y que vaya enlazándose con más instituciones, personas y lugares. Desde luego la conformación de las Sociedades como la nuestra, se caracteriza por tener Comisiones de Trabajo. Hemos de dar apertura a las Comisiones que en principio van a llevar los Ejes que asumimos en esta XIV CONFIBSIG, pero que al irlas desarrollando ya el tiempo nos va a 
ir diciendo como orientarlas de tal manera que esa apertura de Comisiones que nosotros tengamos vaya marcando el rumbo, ese futuro hacia donde nosotros vamos avanzando, siendo hoy unos, mañana otros, en una garantía de que va a continuar el trabajo.

- CONFIBSIG. 1987-2015. También, nosotros somos una Sociedad que ya tiene varias décadas de estar funcionando. La primera data de 1987, habiéndose desarrollado ininterrumpidamente cada dos años. Entonces nos proponemos recuperar toda la organización, las formas de trabajo que hemos tenido en cada una de las Conferencias desde la primera hasta llegar a la XIV CONFIBSIG y ser el portal que va a enlazar con la XV CONFIBSIG. O sea que por allí vamos a entrar, porque dentro de la libertad de organización ellos sabrán darle la organización propia. Por eso es que nosotros estamos hablando que vamos a hallar en el mismo sitio, para conocer cómo es que ha evolucionado, como hemos venido trabajando desde 1987 hasta el 2015.

- Publicaciones CONFIBSIG. También, como resultado de las diferentes conferencias se han dado publicaciones en el tiempo. Eso quiere decir que las diferentes publicaciones van a aparecer para que nosotros las podamos tener a nuestra disposición, bajarlas, y poder darnos cuenta de cuáles son los trabajos importantes y quienes han sido las personas y las líneas que se han trabajado.

- Boletín SIBSIG. Notas Informativas. También hemos puesto allí un Boletín Informativo, Noticias Informativas, de las diferentes actividades que tenemos que hacer que ya no necesariamente tenemos que movilizarnos, sino que a lo mejor podemos hacer, entre la fecha de julio de 2013 a la fecha que se decida para el año 2015, algún evento también virtual, al que todos podamos conectarnos y podamos ver los avances que nosotros tenemos. Entonces, eso se estará informando oportunamente.

- Enlaces de Interés. En el último aspecto nosotros hemos puesto Enlaces de Interés, porque tenemos ya peticiones de cómo nos vamos a enlazar con diferentes organizaciones que afines a nuestro campo y dentro de nuestro campo han pedido que estemos unidos. Y como eso sucede, voy a mencionar dos ejemplos. Un ejemplo es el que me ha presentado la Dra. María Cristina Serafini. Ella menciona la necesidad de que también busquemos una asociación o una forma de comunicación, que ya discutiremos, con lo que es la Sociedad Latinoamericana de Percepción Remota y Sistemas de Información Espacial, SELPER. Entonces yo lo menciono porque va a ser uno de los primeros temas que vamos a discutir. Está también con nosotros el Director General de Catastro y Geografía del Instituto de la Propiedad de Honduras, el Ingeniero 
Fausto Ramírez. El es, como decir en algunos países, el Director del Instituto Geográfico Nacional de Honduras. Los institutos geográficos están vinculados con el Instituto Panamericano de Geografía e Historia, IPGH, y hay compromisos también que asumir con países, concretamente nosotros como Honduras, vamos a empezar a participar en diferentes Comisiones, y entonces la solicitud que el Ingeniero Ramírez ha planteado la recibimos para poder dar el apoyo como Sociedad Iberoamericana de Sistemas de Información Geográfica en la propuesta que se tiene de participar de parte de Honduras y responder al Instituto Panamericano de Geografía e Historia. De esta manera, cuando vayamos expresando nuestras ideas, nuestras formas de actuación a través de esa página que espero se consulte frecuentemente, y que vaya a ser interactiva, pues entonces nosotros vamos a tener la comunicación que esperamos que, cuando se dé el evento de Chile lleguemos con resultados concretos. Yo se que ha sido una jornada larga, posiblemente me podría extender con más cosas, pero al agradecer a la Doctora Serafini y al Ingeniero Ramírez, pues sepan que tienen nuestro compromiso y va ser el primer ejemplo de las formas que vamos a actuar para motivar pues, otras iniciativas de otros lugares y que nuestra Sociedad tenga ya una proyección planetaria.

Punto 7. Cierre de la Sesión. Seguidamente la Presidenta General de la SIBSIG expresó que, con esto no me queda más que cerrar en este momento la Sesión de la Sociedad Iberoamericana de Sistemas de Información Geográfica, para hacerles una nueva Convocatoria en línea en un futuro próximo y que cuando lleguemos a Chile llevemos resultados concretos. Muchas gracias a todos.

\section{Dedicación}

Al presentar el Libro Resúmenes de Conferencias y Comunicaciones de la XIV Conferencia Iberoamericana de Sistemas de Información Geográfica (ISBN: 978-99926-96-59-0), el Comité Local Organizador lo dedicó a la Memoria del Dr. José Antonio Malpica Velasco, participante de la XIV CONFIBSIG, profesor de la Universidad de Alcalá, España, y colaborador de la Maestría en Ordenamiento y Gestión del Territorio de la Facultad de Ciencias Espaciales de la Universidad Nacional Autónoma de Honduras.

Al terminar este PRÓLOGO que contiene la MEMORIA de la XIV Conferencia Iberoamericana de Sistemas de Información Geográfica celebrada en 
Tegucigalpa, Honduras, del 3 al 5 de julio de 2013, dedicamos este trabajo a la Memoria del Dr. Delfino Madrigal Uribe, Presidente General de la SIBSIG 2011-2013, Presidente Ejecutivo de la SIBSIG 2009-2011, conferencista y participante de la XIV CONFIBSIG, profesor de la Facultad de Geografía de la Universidad Autónoma de Estado de México. Su ejemplo, su trabajo y su legado científico permanecerán con nosotros.

MARÍA CRISTINA PINEDADE CARÍAS Decana Facultad Ciencias Espaciales, Universidad Nacional Autónoma de Honduras, Presidenta General SIBSIG 2013-2015

Ciudad Universitaria, Tegucigalpa MDC, Honduras 